\title{
Microbial Predominance and Antimicrobial Resistance in a Tertiary Hospital: A Six-year Retrospective study from Outpatients and Patients Visiting the Emergency Department
}

\author{
CaiFeng Wang \\ Wen Li \\ Xi'an Jiaotong University Medical College First Affiliated Hospital \\ Juanjuan Gao \\ Xi'an Jiaotong University Medical College First Affiliated Hospital \\ Dancheng Zhang \\ Chongqing Medical University \\ Yali Li \\ Xi'an Jiaotong University Medical College First Affiliated Hospital \\ Fang Li \\ Xi'an Jiaotong University Medical College First Affiliated Hospital \\ Jine Lei ( $\square$ lq2640@163.com) \\ Xi'an Jiaotong University Medical College First Affiliated Hospital
}

Xi'an Jiaotong University Medical College First Affiliated Hospital https://orcid.org/0000-0001-7737-4359

\section{Research}

Keywords: Microbial Predominance, Antimicrobial Resistance, Outpatients, Emergency Department

Posted Date: June 1st, 2020

DOI: https://doi.org/10.21203/rs.3.rs-31362/v1

License: (c) (i) This work is licensed under a Creative Commons Attribution 4.0 International License. Read Full License 


\section{Abstract}

Objective: To assessing the characteristics of of microbial species and the antimicrobial resistance in a

Tertiary Hospital with 49 outpatient clinics and emergency department in Northwestern China, of six years.

Methods: A retrospective study was conducted using HIS database of a tertiary hospital between the full-year period of 2013 and 2018.

Antimicrobial susceptibility tests were conducted by automated systems and/or the Kary-Bauer disc diffusion method. Data were analyzed using the WHONET 5.6 software. The Cochran-Armitage test was used to study the trends over the period.

Results: A total of 19,028 specimens were submitted for the laboratory tests of microbiology. Among 49 units, only Emergency Department and Kidney Transplantation Clinic with the number of submission showed a significant increase annually $(P<0.001)$. A total of 3,849 non-repetitive isolates were identified, covering more than 200 species, of which gram-positive cocci accounted for $46.4 \%$ and gram-negative bacilli $45.3 \%$. The methicillin-resistant rates of S. aureus and S. epidermidis were $25.1 \%$ and $74.6 \%$, respectively. The isolates of $60.9 \%$ of $E$. coli and $33.5 \%$ of $K$. pneumonia contained extended spectrum $\beta$ lactamases. Moreover, there were no Staphylococci and Enterococci resistant to linezolid, vancomycin and tigecycline. In addition, the percentages of E. coli, K. pneumonia and P. aeruginosa isolates resistant to carbapenems were low (0.0\%, $1.1 \%$ and $18.7 \%$, respectively).

Conclusion: Vancomycin, linezolid and tigecycline are among the most effective treatment for outpatients with gram-positive infection. Carbapenems are among the most effective for gram-negative infection. There is no significant annual increase of common multidrug resistances.

\section{Introduction}

The increase of using the broad-spectrum antimicrobial treatments leads to microbial resistant to these treatments in which they were originally sensitive [1]. The increased antimicrobial resistance (AMR) limits treatment options and is considered as a global challenge to public health with increased morbidity, mortality and healthcare cost [1]. To accurately estimate the challenge, the surveillance of AMR profiling at local, regional and national levels has been employed to understand the global trends on the type of predominant pathogens and on their respective resistance profiling [2]. In China, the surveillance of AMR is established at the hospital, provincial and national levels. For example, CHINET is one of well-established national surveillance networks for AMR [3-6]. This network recently reported that the frequency of gram-negative bacilli among clinical isolates was over twice higher than that of gram-positive cocci $[3,4]$. Moreover, it is important to recognize that China is a large country with remarkable varieties of microbial occurrences and AMR profiling. For example, Xiao et al. reported that the percentage of MRSA (Methicillin resistant Staphylococcus aureus) in North Central and South Central China is higher than other regions [7]. The geographic variety is likely associated with the regional differences in socioeconomic development [7]. Therefore, the microbial predominance and AMR profiling in a specific region and province is not always consistent with the trend at a national level. Indeed, the surveillance of AMR at the hospital and provincial levels is valuable to complement to the national surveillance. Moreover, the updated AMR profiling at hospital and regional levels is particularly important for the physicians to determine adequate empiric antimicrobial therapy in clinical practice. Therefore, in this study, we analyzed the data from a six-year surveillance of AMR at a tertiary hospital and regional level and attempted to provide insights in both surveillance and clinical practice.

The routine surveillance is mainly derived from hospital-acquired infections and most specimens were taken from inpatient service with severe infections [8]. In contrast, community-acquired infections taken from outpatient service are always under-reported [2, 8]. Therefore, in our study, we tried to bridge the gap and to study the microbial predominance and AMR profiling in outpatient clinics and emergency department in a specific region of Northwestern China and we compared our results with the findings from CHINET.

\section{Methods}

\section{Data Source:}

This study was conducted at the First Affiliated Hospital of Xi'an Jiaotong University, which is located in Northwest China and it is a tertiary class A hospital. This hospital provides medical and surgical care to the residents of Shaanxi Province (total population 37.33 million). An institutional review board approved this project (No: XJTU1AF2017LSK-83) and written informed consent was not obtained since the laboratory tests for microbiology are part of standard care and the record about patient's privacy was excluded prior to analysis. Archived laboratory data between the full-year period of 2013 and 2018 were retrieved from HIS database of the hospital for analysis. A total of 19,028 specimens were collected from 49 outpatient clinics and emergency department. A full list of these clinics was provided in Table 1. The types of the specimens include urine, blood, prostatic fluid, sputum, pleural effusion and ascites, stool, dialysate, pus, secretion, drainage, cerebrospinal fluid and bronchoalveolar lavage fluid.

\section{Isolate identification:}

The clinical specimens were processed based on the recommended microbiology procedures as we previous described [9-12]. Species identification when available was confirmed on colony morphology, conventional biochemical reactions and/or use of an automated system (bioMerieux, Marcy 


\section{Antiobiotic sensitivity:}

Muller-Hinton ( $\mathrm{MH}$ ) agar, $\mathrm{MH}$ agar with $5 \%$ sheep blood Haemophilus test medium (HTM) were purchased from bioMerieux, Marcy l'Etoile, France. The following ATCC strains were used as references: Escherichia coli (ATCC 25922), Pseudomonas aeruginosa (ATCC 27853), Staphylococcus aureus (ATCC 25923), and Enterococcus faecallis (ATCC 29212). An automated system (bioMerieux, Marcy l'Etoile, France) and/or the Kary-Bauer disc diffusion method were used for antimicrobial susceptibility testing following the Clinical Laboratory Standards Institute guideline. The sensitivity breakpoint of cefoperazone/sulbactam referred to that of cefoperazone.

The following agents were tested on isolates from all study years: piperacillin, oxacillin, ampicillin, ampicillin/sulbactam, piperacillin/tazobactam, cefoperazone/sulbactam, cefazolin, cefuroxime, ceftriaxone, cefepime, ciprofloxacin, levofloxacin, moxifloxacin, amikacin, gentamicin, high level gentamicin, tobramycin, aztreonam, erythromycin, clindamycin, vancomycin, linezolid, tetracycline, trimethoprim/sulfamethoxazole and tigecycline.

\section{Statistical analyses:}

Data about the isolates and the susceptibility tests were analyzed using the WHONET 5.6 software provided by World Health Organization. The Cochran-Armitage test was used to study the trends of the specimen numbers submitted from different outpatient clinics, different specimen types and the antibiotic resistance percentage over time. This statistical analysis was performed using R 3.6.1 (R Foundation, Vienna, Austria) and a twosided P-value less than or equal to 0.05 was considered as statistical significance.

\section{Results}

The specimens submitted between different outpatient clinics and emergency department. From 2013 to 2018 , there were 19,028 specimens submitted to the clinical microbiology laboratory from 49 outpatient units. The total number of specimens increased from 2,303 in 2013 to 5,378 in 2018. As we can see from Table 1, the number of specimens submitted from each department showed an increased tendency over the time. The top three units with the largest number of submission were the Emergency Department (30.7\%), Urology (18.9\%) and Nephrology (16.0\%). Moreover, only the Emergency Department and Kidney Transplantation Clinic showed a significant increase on the percentage of specimens submitted annually $(P$ $<0.001)$. Notably, the data from Breast Surgery Clinic were incomplete and therefore they were not included in the analysis. There is no significant difference on annual percentage of specimen submission from the Peritoneal Dialysis Clinic. In contrast, all other departments showed a significant decrease on annual percentage of specimen submission (Table 1). 
Table 1

Annual number and percentage (\%) of the specimens submitted from outpatient clinics and emergency department for the laboratory tests of microbiology from 2013 to 2018

\begin{tabular}{|c|c|c|c|c|c|c|c|c|c|c|c|c|c|c|}
\hline \multirow[b]{2}{*}{ Department } & \multicolumn{2}{|l|}{2013} & \multicolumn{2}{|l|}{2014} & \multicolumn{2}{|l|}{2015} & \multicolumn{2}{|l|}{2016} & \multicolumn{2}{|l|}{2017} & \multicolumn{2}{|l|}{2018} & \multirow{2}{*}{$\begin{array}{l}P \\
\text { value }\end{array}$} & \multirow[t]{2}{*}{ Trend } \\
\hline & $\mathrm{N}$ & $\%$ & $\mathrm{~N}$ & $\%$ & $\mathrm{~N}$ & $\%$ & $\mathrm{~N}$ & $\%$ & $\mathrm{~N}$ & $\%$ & $\mathrm{~N}$ & $\%$ & & \\
\hline Emergency & 469 & 20.4 & 490 & 19.7 & 517 & 21.0 & 1017 & 32.5 & 1046 & 32.0 & 2,306 & 42.9 & $<0.001$ & $\uparrow$ \\
\hline Urology & 400 & 17.4 & 710 & 28.5 & 497 & 20.1 & 426 & 13.6 & 673 & 20.6 & 891 & 16.6 & $<0.001$ & $\downarrow$ \\
\hline Nephrology & 559 & 24.3 & 427 & 17.2 & 491 & 19.9 & 511 & 16.3 & 466 & 14.3 & 581 & 10.8 & $<0.001$ & $\downarrow$ \\
\hline Respiratory & 272 & 11.8 & 211 & 8.5 & 381 & 15.4 & 491 & 15.7 & 300 & 9.2 & 475 & 8.8 & 0.002 & $\downarrow$ \\
\hline Convenient * & 120 & 5.2 & 129 & 5.2 & 134 & 5.4 & 126 & 4.0 & 115 & 3.5 & 160 & 3.0 & $<0.001$ & $\downarrow$ \\
\hline Rheumatology & 150 & 6.5 & 71 & 2.9 & 56 & 2.3 & 68 & 2.2 & 85 & 2.6 & 124 & 2.3 & $<0.001$ & $\downarrow$ \\
\hline $\begin{array}{l}\text { Peritoneal } \\
\text { Dialysis }\end{array}$ & 45 & 2.0 & 91 & 3.7 & 82 & 3.3 & 64 & 2.0 & 69 & 2.1 & 115 & 2.1 & 0.083 & $\begin{array}{l}\text { No } \\
\text { Change }\end{array}$ \\
\hline $\begin{array}{l}\text { Kidney } \\
\text { Transplant }\end{array}$ & 33 & 1.4 & 52 & 2.1 & 25 & 1.0 & 36 & 1.2 & 69 & 2.1 & 143 & 2.7 & 0.0043 & $\uparrow$ \\
\hline Gynecology & 32 & 1.4 & 74 & 3.0 & 80 & 3.2 & 68 & 2.2 & 50 & 1.5 & 53 & 1.0 & $<0.001$ & $\downarrow$ \\
\hline Breast Surgery & $\star \star$ & $\star \star$ & ** & ** & ** & ** & 62 & 2.0 & 103 & 3.2 & 140 & 2.6 & $\star \star$ & ** \\
\hline Other $\star \star \star$ & 223 & 9.7 & 232 & 9.3 & 204 & 8.3 & 258 & 8.3 & 290 & 8.9 & 390 & 7.3 & 0.012 & $\downarrow$ \\
\hline Total & 2,303 & 100.0 & 2,487 & 100.0 & 2,467 & 100.0 & 3,127 & 100.0 & 3,266 & 100.0 & 5,378 & 100.0 & & \\
\hline \multicolumn{15}{|c|}{$\begin{array}{l}\text { * Convenient Clinic helps to meet simple requests of the patients with frequent visits, such as to fill in the prescription and/or to obtain the } \\
\text { regular laboratory tests. }\end{array}$} \\
\hline \multicolumn{15}{|c|}{$\begin{array}{l}\star * \star \text { Other outpatient clinics include Dermatology Clinic, Blood Purification Clinic, Infectious Disease Clinic, Hematology Clinic, Oncology Clinic, } \\
\text { Pediatric Clinic, Chinese Medicine Clinic, Digestive Medicine Clinic, Otolaryngology Clinic, General Surgery Clinic, Orthopedic Surgery Clinic, } \\
\text { Endocrinology Clinic, Ophthalmology Clinic, Thoracic Surgery Clinic, Hepatobiliary Surgery Clinic, Neurology Clinic, Obstetrics Clinic, } \\
\text { Cardiovascular Disease Clinic, Cardiac and Vascular Clinic, Neurosurgery Clinic, Geriatric Surgery Clinic, Oncology Surgery Clinic, Chinese } \\
\text { Medicine Clinic (Xingshan Campus), Neonatal Clinic, Chest Clinic, Burn Plastic Surgery Clinic, Reproductive Medicine Clinic, Cardiovascular } \\
\text { Surgery Clinic, Biotherapy Consultation Clinic, Stomatology Clinic, General Medicine Clinic, Tumor Radiotherapy Clinic, Structural Heart Disease } \\
\text { Clinic, Famous Senior Chinese Medicine Clinic, Peripheral Vascular Clinic, Rehabilitation Clinic (Xingshan Campus), Lugang Obstetrics and } \\
\text { Gynecology Clinic, Pain Clinic and Vascular Surgery Clinic. }\end{array}$} \\
\hline
\end{tabular}

The different types of specimens submitted for the laboratory tests of microbiology. As showed in Table 2, the number of specimens submitted annually among different types of specimens was increased, consistent with the increase of total number of specimen submission each year in Table 1. Sputum, whole blood and pus showed a significant increase on the percentage of specimen submission annually $(P<0.001)$. In contrast, urine, prostatic fluid and other types showed a significant decrease on the percentage of specimen submission annually $(P<0.001)$. Dialysate, peritoneal drainage and pleural effusion and ascites had no significant changes over the period $(P>0.05)$. 
Table 2

Annual number and percentage (\%) of the types of specimens submitted for the laboratory tests of microbiology from outpatients and patients visiting emergency department between 2013 and 2018

\begin{tabular}{|c|c|c|c|c|c|c|c|c|c|c|c|c|c|c|}
\hline & 2013 & & 2014 & & 2015 & & 2016 & & 2017 & & 2018 & & $P$ & Trend \\
\hline $\begin{array}{l}\text { Specimen } \\
\text { type }\end{array}$ & $\mathbf{N}$ & $\%$ & $\mathbf{N}$ & $\%$ & $\mathbf{N}$ & $\%$ & $\mathbf{N}$ & $\%$ & $\mathbf{N}$ & $\%$ & $\mathbf{N}$ & $\%$ & & \\
\hline Urine & 919 & 39.9 & 778 & 31.3 & 805 & 32.6 & 970 & 31.0 & 1,178 & 36.1 & 1,733 & 32.2 & 0.025 & Decreasing \\
\hline Sputum & 395 & 17.2 & 262 & 10.5 & 393 & 15.9 & 672 & 21.5 & 515 & 15.8 & 1,077 & 20.0 & $\begin{array}{l}< \\
0.001\end{array}$ & $\uparrow$ \\
\hline $\begin{array}{l}\text { Whole } \\
\text { blood }\end{array}$ & 231 & 10.0 & 284 & 11.4 & 276 & 11.2 & 511 & 16.3 & 636 & 19.5 & 1,201 & 22.3 & $\begin{array}{l}< \\
0.001\end{array}$ & $\uparrow$ \\
\hline $\begin{array}{l}\text { Prostatic } \\
\text { fluid }\end{array}$ & 248 & 10.8 & 491 & 19.7 & 321 & 13.0 & 220 & 7.0 & 254 & 7.8 & 348 & 6.5 & $<.001$ & $\downarrow$ \\
\hline Pus * & 29 & 1.3 & 33 & 1.3 & 43 & 1.7 & 81 & 2.6 & 142 & 4.3 & 179 & 3.3 & $<.001$ & $\uparrow$ \\
\hline Dialysate & 29 & 1.3 & 85 & 3.4 & 76 & 3.1 & 74 & 2.4 & 96 & 2.9 & 147 & 2.7 & 0.235 & No Change \\
\hline $\begin{array}{l}\text { Peritoneal } \\
\text { drainage }\end{array}$ & 9 & 0.4 & 52 & 2.1 & 45 & 1.8 & 82 & 2.6 & 91 & 2.8 & 48 & 0.9 & 0.709 & No Change \\
\hline $\begin{array}{l}\text { Pleural } \\
\text { effusion } \\
\text { and ascites }\end{array}$ & 8 & 0.3 & 25 & 1.0 & 17 & 0.7 & 52 & 1.7 & 31 & 0.9 & 55 & 1.0 & 0.117 & No Change \\
\hline Others ** & 435 & 18.9 & 477 & 19.2 & 491 & 19.9 & 465 & 14.9 & 323 & 9.9 & 590 & 11.0 & $<.001$ & $\downarrow$ \\
\hline Total & 2,303 & 100.0 & 2,487 & 100.0 & 2,467 & 100.0 & 3,127 & 100.0 & 3,266 & 100.0 & 5,378 & 100.0 & & \\
\hline
\end{tabular}

The microbial growth between different types of specimens. Among the total clinical specimens of 19,028 , a total of 4,719 (24.8\%) were positive for bacterial and fungal growth. The specimen types with highest positive rate were peritoneal drainage $(67 \%$; 219 cases of positive cases from 327 submitted cases), dialysate (52.7\%; 267/507), pus (49.3\%; 250/507) and pleural effusion and ascites (31.9\%; 60/188), followed by urine (28.9\%; $1,843 / 6,383)$, prostatic fluid $(28.1 \% ; 528 / 1,881)$, whole blood $(12.9 \% ; 405 / 3,139)$ and sputum $(9.7 \% ; 323 / 3,314)$.

Common microbial isolates and species. In a total of 4,719 isolates of bacteria and fungi identified, after excluding duplicate isolates obtained from a same patient (each patient was sampled only once), there were a total of 3,849 of non-repetitive isolates. These isolates belong to 211 different species, including 1,786 gram-negative isolates (44.6\%), 1,744 gram-positive isolates (45.3\%), 150 fungus isolates (3.9\%) and 169 isolates with other species (4.4\%). The most frequently identified gram-negative species were Escherichia coli, Klebsiella pneumonia and Pseudomonas aeruginosa, Enterobacter cloacae, Acinetobacter baumannii and Proteus mirabilis. The most frequently identified gram-positive species were Staphylococcus epidermidis, Staphylococcus aureus, Enterococcus faecalis, Staphylococcus haemolyticus, Streptococcus anginosus and Enterococcus faecium. Table 3 shows the number and percentage (\%) of bacterial isolates from the outpatient specimens annually between 2013 and 2018. The percentage of $S$. aureus $(P=0.002)$ and $E$. cloacae $(P=0.033)$ showed a significant increase annually, while $S$. epidermidis $(P<0.001)$ and $S$. haemolyticus $(P<0.001)$ showed a decrease. Candida albicans (78 isolates) and Aspergillus fumigatus (24 isolates) are the major fungi isolated, followed by Aspergillus flavus (9 isolates), Genus of Mucor and Fusarium (9 isolates) and other Candida species (30 isolates). Other rare bacterial isolates included Corynebacterium (109 isolates), Lactobacillus (36 isolates), anaerobic bacteria (13 isolates) as well as Brucella, Eikenella, Actinomyces, Nocardia and Nontuberculous Mycobacterium. Among Corynebacterium, Kroppenstedt Corynebacterium (23 isolates) and Ribbone Corynebacterium (10 isolates) are predominant. 
Table 3

Annual number and percentage (\%) of bacterial isolates from the specimens between 2013 and 2018 from outpatients and patients visiting emergency department

\begin{tabular}{|c|c|c|c|c|c|c|c|c|c|c|c|c|c|c|}
\hline \multirow[b]{2}{*}{ Bacterial Species } & \multicolumn{2}{|c|}{2013} & \multicolumn{2}{|c|}{2014} & \multicolumn{2}{|c|}{2015} & \multicolumn{2}{|c|}{2016} & \multicolumn{2}{|c|}{2017} & \multicolumn{2}{|c|}{2018} & \multirow{2}{*}{$\begin{array}{l}P \\
\text { value }\end{array}$} & \multirow[t]{2}{*}{ Trend } \\
\hline & $\mathbf{N}$ & $\%$ & $\mathbf{N}$ & $\%$ & $\mathbf{N}$ & $\%$ & $\mathbf{N}$ & $\%$ & $\mathbf{N}$ & $\%$ & $\mathbf{N}$ & $\%$ & & \\
\hline \multicolumn{15}{|l|}{ Gram-negative } \\
\hline Escherichia coli & 134 & 70.2 & 149 & 66.8 & 128 & 61.0 & 138 & 53.5 & 218 & 60.2 & 268 & 56.9 & 0.471 & $\begin{array}{l}\text { No } \\
\text { Change }\end{array}$ \\
\hline Klebsiella pneumonia & 21 & 11.0 & 24 & 10.8 & 14 & 6.7 & 29 & 11.2 & 38 & 10.5 & 56 & 11.9 & 0.400 & $\begin{array}{l}\text { No } \\
\text { Change }\end{array}$ \\
\hline $\begin{array}{l}\text { Pseudomonas } \\
\text { aeruginosa }\end{array}$ & 9 & 4.7 & 19 & 8.5 & 11 & 5.2 & 18 & 7.0 & 18 & 5.0 & 32 & 6.8 & 0.760 & $\begin{array}{l}\text { No } \\
\text { Change }\end{array}$ \\
\hline Enterobacter cloacae & 4 & 2.1 & 3 & 1.3 & 1 & 0.5 & 9 & 3.5 & 12 & 3.3 & 22 & 4.7 & 0.033 & $\uparrow$ \\
\hline Acinetobacter baumannii & 6 & 3.1 & 5 & 2.2 & 7 & 3.3 & 7 & 2.7 & 9 & 2.5 & 14 & 3.0 & 0.870 & $\begin{array}{l}\text { No } \\
\text { Change }\end{array}$ \\
\hline Proteus mirabilis & 3 & 1.6 & 6 & 2.7 & 5 & 2.4 & 9 & 3.5 & 11 & 3.0 & 7 & 1.5 & 0.985 & $\begin{array}{l}\text { No } \\
\text { Change }\end{array}$ \\
\hline Others* & 14 & 7.3 & 17 & 7.6 & 44 & 21.0 & 48 & 18.6 & 56 & 15.5 & 72 & 15.3 & & \\
\hline Total & 191 & 100.0 & 223 & 100.0 & 210 & 100.0 & 258 & 100.0 & 362 & 100.0 & 471 & 100.0 & & \\
\hline \multicolumn{15}{|l|}{ Gram-positive } \\
\hline $\begin{array}{l}\text { Staphylococcus } \\
\text { epidermidis }\end{array}$ & 37 & 18.1 & 81 & 28.1 & 37 & 20.4 & 29 & 12.7 & 45 & 18.0 & 39 & 11.3 & $\begin{array}{l}< \\
0.001\end{array}$ & $\downarrow$ \\
\hline Staphylococcus aureus & 12 & 5.9 & 20 & 6.9 & 20 & 11.0 & 45 & 19.7 & 50 & 20.0 & 80 & 23.3 & 0.002 & $\uparrow$ \\
\hline Enterococcus faecalis & 21 & 10.3 & 33 & 11.5 & 20 & 11.0 & 30 & 13.2 & 33 & 13.2 & 56 & 16.3 & 0.984 & $\begin{array}{l}\text { No } \\
\text { Change }\end{array}$ \\
\hline $\begin{array}{l}\text { Staphylococcus } \\
\text { haemolyticus }\end{array}$ & 34 & 16.7 & 39 & 13.5 & 22 & 12.2 & 12 & 5.3 & 11 & 4.4 & 20 & 5.8 & $\begin{array}{l}<.001 \\
0.001\end{array}$ & $\downarrow$ \\
\hline Streptococcus anginosus & 5 & 2.5 & 16 & 5.6 & 16 & 8.8 & 17 & 7.5 & 20 & 8.0 & 28 & 8.1 & 0.556 & $\begin{array}{l}\text { No } \\
\text { Change }\end{array}$ \\
\hline Enterococcus faecium & 9 & 4.4 & 7 & 2.4 & 10 & 5.5 & 17 & 7.5 & 19 & 7.6 & 32 & 9.3 & 0.163 & $\begin{array}{l}\text { No } \\
\text { Change }\end{array}$ \\
\hline Others** & 86 & 42.2 & 92 & 31.9 & 56 & 30.9 & 78 & 34.2 & 72 & 28.8 & 89 & 25.9 & & \\
\hline Total & 204 & 100.0 & 288 & 100.0 & 181 & 100.0 & 228 & 100.0 & 250 & 100.0 & 344 & 100.0 & & \\
\hline \multicolumn{15}{|c|}{$\begin{array}{l}\text { * Other gram-negative bacteria mainly include Enterobacteria, Pseudomonas, Proteus, Citrobacter, Haemophilus, Neisseria, Bacteroides, } \\
\text { Aeromonas and Morgan Bacteria. }\end{array}$} \\
\hline
\end{tabular}

The antimicrobial resistance profile of the common pathogens. 1) Staphylococcus spp. Our study showed that all of $S$. aureus isolates in our community are resistance to penicillin (Table 4). However, many $S$. aureus strains, while resistant to penicillin, remain susceptible to penicillinasestable penicillins, such as oxacillin. Strains that are resistant to oxacillin and methicillin are historically termed methicillin-resistant $S$. aureus (MRSA) [13]. Our study showed that the percentage of MRSA isolates is $25.1 \%$ during the studied period (Table 4) without significant changes annually (Table 6). In contrast, CHINET reported that the prevalence of MRSA was decreased from $69.0 \%$ in 2005 to $35.2 \%$ in 2017 [3]. The difference between CHINET and our study is likely due to the prevalence of MRSA in community acquired infections is lower over the period of our study, suggesting the infiltration of MRSA isolates from hospitals to community is very limited. 
Table 4

Antimicrobial resistance percentage (\%) of top four gram-positive cocci among common antimicrobial agents between 2013 and 2018 from outpatients and patients visiting emergency department

\begin{tabular}{|c|c|c|c|c|}
\hline \multirow[t]{2}{*}{ Antimicrobial Agent } & Staphylococcus aureus & Staphylococcus epidermidis & Enterococcus faecalis & Enterococcus faecium \\
\hline & $(n=227) *$ & $(n=268) *$ & $(n=193) *$ & $(n=94) *$ \\
\hline \multicolumn{5}{|l|}{ Penicillins } \\
\hline Penicillin G & 100.0 & 96.6 & 0.0 & 94.7 \\
\hline Oxacillin & 25.1 & 74.6 & ** & ** \\
\hline Ampicillin & ** & ** & 0.0 & 94.7 \\
\hline \multicolumn{5}{|l|}{ Fluoroquinolones } \\
\hline Ciprofloxacin & 17.6 & 32.1 & 23.8 & 96.8 \\
\hline Levofloxacin & 17.6 & 40.7 & 22.8 & 94.7 \\
\hline Moxifloxacin & 6.6 & 7.5 & $\star *$ & $\star \star$ \\
\hline \multicolumn{5}{|l|}{ Aminoglycosides } \\
\hline Gentamicin & 23.3 & 8.6 & ** & $\star \star$ \\
\hline Gentamicin HLAR & ** & $\star \star$ & 0.0 & 0.0 \\
\hline \multicolumn{5}{|l|}{ Others } \\
\hline Erythromycin & 70.5 & 82.1 & 57 & 90.4 \\
\hline Clindamycin & 41.4 & 22 & $\star *$ & $\star \star$ \\
\hline Vancomycin & 0.0 & 0.0 & 0.0 & 0.0 \\
\hline Linezolid & 0.0 & 0.0 & 0.0 & 0.0 \\
\hline Tetracycline & 41.4 & 20.1 & 66.8 & 45.7 \\
\hline Trimethoprim/sulfamethoxazole & 29.5 & 64.6 & 0.0 & 0.0 \\
\hline Tigecycline & 0.0 & 0.0 & 0.0 & 0.0 \\
\hline
\end{tabular}


Table 6

Annual multidrug resistance percentage (R\%) of bacterial isolates from the specimens between 2013 and 2018 from outpatients and patients visiting emergency department

\begin{tabular}{|c|c|c|c|c|c|c|c|c|c|}
\hline \multirow[t]{2}{*}{ Bacterial Species } & Years & 2013 & 2014 & 2015 & 2016 & 2017 & 2018 & \multirow[t]{2}{*}{$P$ value } & \multirow[t]{2}{*}{ Trend } \\
\hline & Multidrug resistance targets & $\mathrm{R} \%$ & $\mathbf{R} \%$ & $\mathrm{R} \%$ & $\mathbf{R} \%$ & $\mathrm{R} \%$ & $\mathrm{R} \%$ & & \\
\hline \multirow[t]{2}{*}{ Escherichia coli } & ESBLs* & 62.6 & 59.6 & 57.4 & 59.3 & 56.8 & 59.4 & 0.586 & No Change \\
\hline & $\mathrm{CRE}^{\star \star}$ & 0.0 & 0.0 & 0.0 & 0.0 & 0.0 & 0.0 & - & No Change \\
\hline \multirow[t]{2}{*}{ Klebsiella pneumoniae } & ESBLs* & 29.2 & 40.5 & 20.0 & 30.0 & 39.3 & 32.5 & 0.935 & No Change \\
\hline & $\mathrm{CRE}^{\star \star}$ & 0.0 & 0.0 & 0.0 & 0.0 & 1.8 & 1.3 & 0.443 & No Change \\
\hline Staphylococcus aureus & MRSA $^{\#}$ & 53.8 & 27.6 & 25.9 & 25 & 25 & 19.2 & 0.191 & No Change \\
\hline Staphylococcus epidermidis & MRSE $\#$ & 88.1 & 83.3 & 78.7 & 94.1 & 72.7 & 75.6 & 0.213 & No Change \\
\hline \multicolumn{10}{|c|}{ *ESBLs (Extended spectrum $\beta$ lactamases): ESBLs multidrug resistance was evaluated by using ceftriaxone. } \\
\hline \multicolumn{10}{|c|}{${ }^{\star *}$ CRE (Carbapenem - resistant enterobacteriaceae): CRE multidrug resistance was evaluated by using imipenem and meropenem. } \\
\hline \multicolumn{10}{|c|}{ \#MRSA (Methicillin resistant $S$. aureus): MRSA multidrug resistance was evaluated by using oxacillin. } \\
\hline
\end{tabular}

As showed in Table 4, less than $30 \%$ of $S$. aureus isolates were resistance to trimethoprim/sulfamethoxazole (29.5\%), gentamicin (23.3\%), ciprofloxacin (17.6\%), levofloxacin (17.6\%) and moxifloxacin (6.6\%), indicating a minor resistance to fluoroquinolones and aminoglycosides. Whereas more than $30 \%$ of $S$. aureus isolates were resistance to erythromycin (70.5\%), clindamycin (41.4\%) and tetracycline (41.4\%).

Similar to $S$. aureus, nearly all of $S$. epidermidis isolates (96.6\%) in our community acquired infections are resistance to penicillin (Table 4). Strains of $S$. epidermidis that are resistant to oxacillin and methicillin are historically termed methicillin-resistant S. epidermidis (MRSE). Our study showed that the percentage of MRSE isolates was $74.6 \%$ during the study period (Table 4) without significant changes annually (Table 6 ). Less than $30 \%$ of S. epidermidis isolates were resistance to clindamycin (22.0\%), tetracycline (20.1\%), gentamicin (8.6\%) and moxifloxacin (7.5\%). Whereas more than $30 \%$ of S. epidermidis isolates were resistance to erythromycin (82.1\%), compound sulfamethoxazole (64.6\%), levofloxacin (40.7\%) and ciprofloxacin (32.1\%). These findings indicate a medium resistance of S. epidermidis to fluoroquinolones and a minor resistance to aminoglycosides. More important, all of $S$. epidermidis and $S$. aureus isolates collected from our study were sensitive to vancomycin, linezolid and tigecycline.

2) Enterococcus spp. Among Enterococcus species, two major species (Enterococcus faecalis and Enterococcus faecium) are particularly pathogenic for humans. As showed in Table 4, although the number of isolates of E. faecalis was remarkably higher than that from $E$. faecium (193 isolates vs 94$)$, E. faecalis isolates were less resistance to most antibiotics compared to $E$. faecium isolates, including ampicillin ( $0.0 \%$ vs $94.7 \%)$, erythromycin ( $57.0 \%$ vs $90.4 \%)$, ciprofloxacin (23.8\% vs $96.8 \%)$, penicillin (0.0\% vs $94.7 \%$ ) and levofloxacin (22.8\% vs $94.7 \%)$; the only exception was tetracycline (66.8\% vs $45.7 \%)$. Furthermore, all of E. faecalis and E. faecium isolates collected from our study were sensitive to linezolid, tigecycline and vancomycin.

3) Enterobacteriaceae. Enterobacteriaceae, including E. coli and $K$. pneumonia, produces extended-spectrum $\beta$-lactamases (ESBLs). ESBLs are a group of $\beta$-lactamases, which share the ability to hydrolyze $\beta$-lactam antibiotics, such as cephalosporins [14]. By using ceftriaxione as substrate, our study suggested that $60.9 \%$ of $E$. coli isolates and $33.5 \%$ of $K$. pneumonia contained ESBLs (Table 5). Consistent with ESBLs results, the similar trend was observed for the percentage of $E$. coli and $K$. pneumonia isolates resistant to other cephalosporins: cefepime (33.0\% vs $5.9 \%)$, cefuroxime ( $80.7 \%$ vs $23.5 \%)$, ceftazidime ( $54.5 \%$ vs $17.6 \%$ ) and cefazolin (79.5\% vs $17.6 \%$ ); the exception is cefotetan (5.7\% vs $0.0 \%)($ Table 5$)$. Less than $30 \%$ of E. coli isolates were resistance to tobramycin (21.6\%), cefoperazone/sulbactam (8.4\%), piperacillin/tazobactam (8.0\%) and amikacin (2.3\%). More than $30 \%$ of E. coli isolates were resistance to ampicillin (93.2\%), piperacillin (86.4\%), ciprofloxacin (80.7\%), levofloxacin (76.1\%), ampicillin/shubatan (70.5\%), trimethoprim/sulfamethoxazole (62.5\%), aztreonam (60.2\%) and gentamicin (39.8\%), indicates a high resistance to penicillins and fluoroquinolones. All of $E$. coli isolates were sensitive to imipenem and meropenem. 
Table 5

Antimicrobial resistance percentage (\%) of top three gram-negative bacilli among common antimicrobial agents between 2013 and 2018 from outpatients and patients visiting emergency department

\begin{tabular}{|c|c|c|c|}
\hline \multirow[t]{2}{*}{ Antimicrobial Agent } & Escherichia coli & Klebsiella pneumoniae & Pseudomonas aeruginosa \\
\hline & $(n=1035)^{\star}$ & $(n=182)^{*}$ & $(n=107) *$ \\
\hline \multicolumn{4}{|l|}{ Penicillins } \\
\hline Piperacillin & 86.4 & 17.6 & 14.9 \\
\hline Ampicillin & 93.2 & $-* \star$ & $-\star \star \star$ \\
\hline \multicolumn{4}{|l|}{ Cephems } \\
\hline Cefazolin & 79.5 & 17.6 & $-* *$ \\
\hline Cefuroxime & 80.7 & 23.5 & $-\star *$ \\
\hline Ceftriaxone & 60.9 & 33.5 & $-* *$ \\
\hline Ceftazidime & 54.5 & 17.6 & 15.0 \\
\hline Cefepime & 33.0 & 5.9 & 7.5 \\
\hline Cefotetan & 5.7 & 0.0 & $-\star \star$ \\
\hline \multicolumn{4}{|l|}{$\beta$-lactam combination agents } \\
\hline Ampicillin/Shubatan & 70.5 & 23.5 & $-\star *$ \\
\hline Piperacillin/Tazobactam & 8.0 & 0.0 & 7.5 \\
\hline Cefoperazone/sulbactam & 8.4 & 0.0 & 10.3 \\
\hline \multicolumn{4}{|l|}{ Fluoroquinolones } \\
\hline Ciprofloxacin & 80.7 & 11.8 & 0.0 \\
\hline Levofloxacin & 76.1 & 11.8 & 0.0 \\
\hline \multicolumn{4}{|l|}{ Aminoglycosides } \\
\hline Amikacin & 2.3 & 0.0 & 7.5 \\
\hline Gentamicin & 39.8 & 5.9 & 7.5 \\
\hline Tobramycin & 21.6 & 0.0 & 7.5 \\
\hline \multicolumn{4}{|l|}{ Others } \\
\hline Aztreonam & 60.2 & 5.9 & 22.4 \\
\hline Imipenem & 0.0 & 1.1 & 18.7 \\
\hline Meropenem & 0.0 & 1.1 & 18.7 \\
\hline Trimethoprim/sulfamethoxazole & 62.5 & 29.4 & $-\star \star *$ \\
\hline \multicolumn{4}{|c|}{ * $\mathrm{n}$ represents the number of non-repetitive isolates for each species. } \\
\hline$\star \star$ indicates that the result is not $a$ & ¡lable. & & \\
\hline
\end{tabular}

As showed in Table 5, less than $30 \%$ of $K$. pneumonia isolates were resistance to compound sulfamethoxazole (29.4\%), ampicillin/shubatan (23.5\%), piperacillin (17.6\%), ciprofloxacin (11.8\%), levofloxacin (11.8\%), aztreonam (5.9\%), gentamicin (5.9\%), imipenem (1.1\%) and meropenem (1.1\%), indicates a minor resistance to these common antibiotics, such as penicillins and fluoroquinolones. All of $K$. pneumonia isolates were sensitive to amikacin, piperacillin/tazobactam, cefoperazone/sulbactam, cefotetan and tobramycin.

4) Non-fermentative bacteria. All of $P$. aeruginosa isolates were sensitive to fluoroquinolones, including ciprofloxacin and levofloxacin. The resistance of $P$. aeruginosa isolates to other antibiotics was all less than 30\%: aztreonam (22.4\%), metopenem (18.7\%), imipenem (18.7\%), ceftazidime (15.0\%), piperacillin (14.9\%), cefoperazone/sulbactam (10.3\%), amikacin (7.5\%), piperacillin/tazobactam (7.5\%), gentamicin (7.5\%), cefepime (7.5\%) and tobramycin (7.5\%), suggesting a minor resistance to these common antibiotics.

\section{Discussion}


In this study we reported the microbial predominance and AMR profiling in a tertiary hospital using a six-year retrospective data from outpatient clinics and emergency department within a specific region of Northwestern China. We will discuss our findings in the following five aspects.

1. The medical value to study the distribution of specimen submitted between different outpatient clinics and emergency department. Based on our literature search, there is little publication to compare the number and percentage of specimens submitted to the clinical microbiology laboratory between different outpatient clinics. Different from other countries, Chinese patients can directly visit the specialty services without the need of the referral from the primary care. Our study showed a trend of increased number on specimen submission in each clinic (Table 1 ), which is likely associated with the increased population in the communities. The Emergency Department, Urology and Nephrology were among the top three clinics that submitted specimens for microbiological studies. Urinary tract infection has been considered the major reason for outpatients to visit Urology and Nephrology [15] and is also one of the major concerns for emergency room [16]. More important, our study revealed that only two departments including the Emergency Department and Kidney Transplantation Clinic showed a significant increase on the percentage of specimen submitted annually $(P<0.001)$, which indicates that these two departments, particularly the Emergency Department [17], are in the front line of infection control. The increased importance of the Emergency Department is likely associated with enhanced medical functions of the emergency service; there is a trend for the Emergency Department to handle more previous inpatient cares. The increased importance of Kidney Transplantation Department indicated that Kidney Transplantation is becoming a mainstream medical service and repeated infection after kidney transplantation is an urgent medical issue in outpatient clinics [18].

2. Relatively lower ratio of gram-negative isolates in our community acquired infections. Among 3,849 non-repetitive isolates, the ratio of gramnegative vs gram-positive is nearly $1: 1$ (45.3\% vs $46.4 \%$ ) in our study. In contrast, CHINET report the ratio is nearly $2: 1$ with approximately $70 \%$ of gram-negative bacteria $[3,4]$. Since CHINET data was largely derived from inpatient service, the relatively lower ratio of gram-negative isolates in our community acquired infections is likely due to the difference between outpatient and inpatient service. The studies from US hospitals report that gram-negative bacteria are more common in cases of ventilator-associated pneumonia and urinary tract infection [19] and are a dominant type of infection at intensive care units [20]. These results suggest a likely association between the higher ratio of gram-negative infection and the inpatient experiencing invasive medical devices and surgical procedure. Indeed, the gram-negative bacteria contain highly efficient mechanism of antibiotic drug resistance [21]. By comparing Tables 4 and 5 in our study, gram-negative bacteria, particularly E. coli, appear more resistant to the antibiotics and require more complicated therapeutic regimen. Overall, relatively lower ratio of gram-negative isolates is likely a general feature in outpatient service.

3. Vancomycin, linezolid and tigecycline are among the most effective treatment for patients with gram-positive infection in our community. Vancomycin, linezolid and tigecycline have been used to treat multidrug resistance bacteria in the community [22]. In our study, Staphylococci and Enterococci (Table 4) are $100 \%$ sensitive to vancomycin and linezolid, which is in general consistent with the findings from CHINET. For example, CHINET surveillance in 2018 showed that Staphylococci isolated from blood, urine, lower respiratory tract and cerebrospinal fluid were 100\% sensitive to vancomycin and linezolid except less than $1 \%$ of Staphylococci isolated from blood resist to linezolid [4]. Moreover, less than $5 \%$ of $E$. faecium and less than 1\% of E. faecalis were vancomycin-resistant in CHINET surveillance from 2005 to 2017 [3]. These findings indicate that vancomycin and linezolid remains the most effective treatment for gram-positive infection for both hospital and community settings.

In our study, Staphylococci and Enterococci (Table 4) are also 100\% sensitive to tigecycline, an expanded broad-spectrum intravenous glycylcycline antibiotic. This finding supports tigecycline as a reserve for multidrug resistance bacterial infection in community settings.

4. Carbapenems are among the most effective treatment for patients with gram-negative infection in our community. Table 5 showed that higher resistance of gram-negative pathogens, particularly E. coli, to the cephalosporins and quinolones in this study. To overcome the clinical challenge, carbapenems such as imipenem and meropenem have been reserved for these multidrug-resistant bacterial infections [23]. Table 5 also showed that the percentages of E. coli, K. pneumonia and P. aeruginosa isolates resistant to imipenem were $0.0 \%, 1.1 \%$ and $18.7 \%$, respectively. Moreover, the annual trend of resistant percentage of E. coli and K. pneumonia to carbapenems is not significant increased (Table 6). These findings support that carbapenems as a reserve for multidrug resistance bacterial infection in community settings.

5. There is no significant increase of multidrug-resistant bacteria observed in our community settings. Tables 4 and 5 showed higher resistance percentage between certain bacteria and antibiotics, such as methicillin-resistant gram-positive pathogens (MRSA and MRSE) and extended spectrum $\beta$ lactamases (ESBLs)-resistant gram-negative pathogens. By using ceftriaxone and oxacillin, Table 6 showed the annual change of the resistance rate of multidrug-resistant bacteria in recent years. Should has a significant increase of resistance rate been observed, it would require an immediate intervention to investigate the reason. Our results suggest that there is no significant increase of multidrug-resistant bacteria, indicating a reasonable prescription of antibiotics in our outpatient and emergency services and a successful physician education on the prevention of antibiotic resistance.

Our study has several limitations. For example, this was a single-center retrospective observational study. We must be cautious when translating our results to other hospitals. However, the quality of clinical sampling procedure and techniques were in general better controlled in a single center than multiple centers. They also allow us to obtain a representative specimen in a large number of clinical departments throughout the hospital and, when repeated periodically, show the main trends. Moreover, a single-center study when appropriately carried out is useful and inexpensive surveilling tool to reflect the regional situation of prevailing microorganisms and their resistance to antimicrobials. Second, outpatient surveillance is 
an important tool to study the community-acquired infection. We must realize that not all infection of outpatient and patients visiting emergency department derive from the community. This is the reason we only used the term of "community settings" in this article since the patients directly came from the free-living community to seek the medical care in our outpatient and emergency services.

\section{Declarations}

\section{Ethics Approval and Consent to Participants}

This project was approved by the Institutional Review Board of the First Affiliated Hospital of Xi'an Jiaotong Unviersity (No: XJTU1AF2017LSK-83) but the written informed consent can not obtained since the laboratory tests for microbiology are part of standard care and the record about patient's privacy was removed prior to data analysis.

\section{Availability of Data and Material}

All the data are available upon request.

\section{Competing Interests}

On behalf of all authors, the corresponding author states that there is no conflict of interest.

\section{Consent for Publication}

All authors have agreed to publish the results.

\section{Funding}

This study was funded by the Shaanxi Key Science and Technology Project (To JL, No: 2017SF-198).

\section{Authors' Contributions}

Caifeng Wang, study design, funding application, study manager, and manuscript writing; Jine Lei, laboratory analysis; Wen Li ang Yali Li, data entry ; Juanjuan Gao, Statistical analyses of data; Dancheng Zhang and Fang Li, data analysis.

\section{References}

1. Roope LSJ, Smith RD, Pouwels KB, Buchanan J, Abel L, Eibich P, et al. The challenge of antimicrobial resistance: What economics can contribute. Science. 2019;364:eaau4679.

2. Croxall G, Hale J, Weston V, Manning G, Cheetham P, Achtman M, et al. Molecular epidemiology of extraintestinal pathogenic Escherichia coli isolates from a regional cohort of elderly patients highlights the prevalence of ST131 strains with increased antimicrobial resistance in both community and hospital care settings. J Antimicrob Chemother. 2011;66:2501-8.

3. Hu F, Zhu D, Wang F, Wang M. Current Status and Trends of Antibacterial Resistance in China. Clin Infect Dis. 2018;67:128-S34.

4. Hu F, Guo Y, Yang Y, Zheng Y, Wu S, Jiang X, et al. Resistance reported from China antimicrobial surveillance network (CHINET) in 2018. European journal of clinical microbiology infectious diseases: official publication of the European Society of Clinical Microbiology. 2019;38:2275-81.

5. Hu FP, Guo Y, Zhu DM, Wang F, Jiang XF, Xu YC, et al. Resistance trends among clinical isolates in China reported from CHINET surveillance of bacterial resistance, 2005-2014. Clinical microbiology and infection: the official publication of the European Society of Clinical. Microbiology Infectious Diseases. 2016;22(Suppl 1):9-14.

6. Yin D, Wu S, Yang Y, Shi Q, Dong D, Zhu D, et al. Results from the China Antimicrobial Surveillance Network (CHINET) in 2017 of the In Vitro Activities of Ceftazidime-Avibactam and Ceftolozane-Tazobactam against Clinical Isolates of Enterobacteriaceae and Pseudomonas aeruginosa. Antimicrobial agents and chemotherapy 2019;63.

7. Xiao Y, Wei Z, Shen P, Ji J, Sun Z, Yu H, et al. Bacterial-resistance among outpatients of county hospitals in China: significant geographic distinctions and minor differences between central cities. Microbes Infect. 2015;17:417-25.

8. World Health O. Antimicrobial resistance: global report on surveillance. Geneva: World Health Organization; 2014. 
9. Li S, Guo Y, Zhao C, Chen H, Hu B, Chu Y, et al. In vitro activities of tedizolid compared with other antibiotics against Gram-positive pathogens associated with hospital-acquired pneumonia, skin and soft tissue infection and bloodstream infection collected from 26 hospitals in China. $J$ Med Microbiol. 2016;65:1215-24.

10. Han L, Lei J-e, Wang X, Guo L-t, Kang Q-y, He L, et al. Septicemia Caused by < span class="named-content genus-species" id="named-content1 ">Leifsonia aquatica</span > in a Healthy Patient after Retinal Reattachment Surgery. Journal of Clinical Microbiology 2013;51:3886-8.

11. Zhao C, Zhang F, Chu Y, Liu Y, Cao B, Chen M, et al. Phenotypic and Genotypic Characteristic of Invasive Pneumococcal Isolates from Both Children and Adult Patients from a Multicenter Surveillance in China 2005-2011. PLOS ONE. 2013;8:e82361.

12. Lei Je, Han S, Wu W, Wang X, Xu J, Han L. Extensively drug-resistant < em > Acinetobacter baumannii</em > outbreak cross-transmitted in an intensive care unit and respiratory intensive care unit. American Journal of Infection Control 2016;44:1280-4.

13. https://gram.cdc.gov/hai/settings/lab/lab_mrsa.html.

14. Dimitriu T, Medaney F, Amanatidou E, Forsyth J, Ellis RJ, Raymond B. Negative frequency dependent selection on plasmid carriage and low fitness costs maintain extended spectrum $\beta$-lactamases in Escherichia coli. Sci Rep. 2019;9:17211.

15. Fiore DC, Fox C-L. Urology and nephrology update: recurrent urinary tract infection. FP Essent. 2014;416:30-7.

16. Dubbs SB, Sommerkamp SK. Evaluation and Management of Urinary Tract Infection in the Emergency Department. Emerg Med Clin North Am. 2019;37:707-23.

17. Mistry RD, Dayan PS, Kuppermann N. The Battle Against Antimicrobial Resistance: Time for the Emergency Department to Join the Fight. JAMA Pediatrics. 2015;169:421-2.

18. Chen Y-X, Li R, Gu L, Xu K-Y, Liu Y-Z, Zhang R-W. Risk factors and etiology of repeat infection in kidney transplant recipients. Medicine. 2019;98:e17312.

19. Antimicrobial-Resistant Pathogens Associated With Healthcare-Associated Infections: Annual Summary of Data Reported to the National Healthcare Safety Network at the Centers for Disease Control and Prevention, 2006-2007

Hidron Al, Edwards JR, Patel J, Horan TC, Sievert DM, Pollock DA, et al. Antimicrobial-Resistant Pathogens Associated With HealthcareAssociated Infections: Annual Summary of Data Reported to the National Healthcare Safety Network at the Centers for Disease Control and Prevention, 2006-2007. Infection Control Hospital Epidemiology 2015;29:996-1011.

20. Weinstein RA, Gaynes R, Edwards JR, System NNIS. Overview of Nosocomial Infections Caused by Gram-Negative Bacilli. Clin Infect Dis. 2005;41:848-54.

21. Peleg AY, Hooper DC. Hospital-Acquired Infections Due to Gram-Negative Bacteria. N Engl J Med. 2010;362:1804-13.

22. van Duin D, Paterson DL. Multidrug-Resistant Bacteria in the Community: Trends and Lessons Learned. Infect Dis Clin North Am. 2016;30:37790.

23. Hawkey PM, Livermore DM. Carbapenem antibiotics for serious infections. BMJ: British Medical Journal. 2012;344:e3236. 